\title{
Study on Dependency Levels in the Oncological Patient with Respiratory Symptomatology
}

\section{Fernandez Rodrigue EJ* and Sanchez Gomez C \\ Department of Occupational Therapy, University of Salamanca, Spain}

*Corresponding author: Fernandez Rodrigue EJ, Department of Occupational Therapy, University of Salamanca, Spain; Email: edujfr@usal.es

\section{Research Article \\ Volume 2 Issue 3}

Received Date: September 10, 2019

Published Date: September 27, 2019

DOI: $10.23880 /$ aphot- 16000138

\section{Abstract}

The incidence of cancer in Spain is increasing (annually 247,771 new cases diagnosed). Survival of oncological patients at 5 years of 53\%. Main problem, the quality of that survival. Important conditioning factor, the levels of dependence of oncological patients in active treatment. The main objective was to evaluate the level of dependence of oncological patients with dyspnea.

Methodology: Cross-sectional prospective observational study with non-probabilistic sampling of accidental allocation. The sample was recruited from the Medical Oncology Service of the University Hospital of Salamanca (CAUSA). We proceeded to refer patients to the occupational therapy program with the consequent inclusion of these in the study. After obtaining the informed consent signed by the subjects, the evaluation was proceeded. The Lawton-Brody scale (AIVD), and the Barthel index (ABVD). In addition, a record sheet for sociodemographic and clinical (age, gender and anatomopathological diagnosis) was designed.

Results: Study includes the sample size of 180 participants.. Average age of $68.48( \pm 10,513)$ years, of which $52.8 \%$ men, by $47.2 \%$ women ( 85 women and 95 men). Of which $22.8 \%$ had breast diagnosis, $31.7 \%$ pulmonary and $45.6 \%$ related to the digestive system. Regarding dependence levels, Lawton-Brody scale, mean score of $4.31( \pm 1.832)$ points (moderate dependency levels); Barthel index, mean score of $42.53( \pm 2,789)$ points (moderate dependency level). Poor scores in lung anatomopathological diagnosis. Strong and statistically significant correlation between ABVD and AIVD ( $r=0.803$, for $\mathrm{p}<0.05)$, and significant correlation $(\mathrm{r}=-0.403$, for $\mathrm{p}<0.001)$ between age and the performance of IADL.

Conclusion: Oncological patients with respiratory symptoms present moderate levels of dependence.

Keywords: Functionality; Activities of Daily Life; Cancer; Occupational Therapy

Abbreviations: GLOBOCAN: Global Cancer Observatory; ADLs: Activities of Daily Living; MRC: Medical Research Council. 


\section{Annals of Physiotherapy \& Occupational Therapy}

\section{Introduction}

Analyzing the data present in the Global Cancer Observatory (GLOBOCAN), currently the prevalence in cancer is almost three cases per thousand inhabitants worldwide, being the second leading cause of death in the world (approximately 13\% of the total). The tumors that cause the most deaths each year are cancers of the lung, stomach, liver, colon and breast [1].

According to the Spanish Society of Medical Oncology, in the latest data published in 2014, 247,771 new cases of cancer are diagnosed each year $(148,827$ in men and 98,944 in women) and more than 1.5 million people are affected by the disease [2].

If we speak in global terms, cancer continues to be one of the leading causes of morbidity and mortality, with approximately 14 million new cases worldwide in 2012 [3].

Cancer mortality in Spain, in 2014, was 106,039 deaths $(65,019$ cases in men and 41,020 in women).

From the cancer or its treatments, a series of associated symptoms that decrease in these, their ability to function normally in their daily lives. Some of these symptoms may be asthenia, anxiety or dyspnea. In the latter, respiratory infections found one of the main causes of death in the progressive cancer patient [2]. Dyspnea, together with cough and hemoptysis, in addition to being the most frequent symptoms in these type of patients, that cause more anxiety to both the sick and their care givers [3].

In some patients with advanced cancer, this dyspnea may represent a clinical sign of the final phase of the disease [4]. Approximately $41 \%$ of hospice patients have the symptom and the $46 \%$ of them describe moderate or severe intensity in May.

As in the case of another symptom, such as pain, because it is a subjective sensation, only the patient can judge exactly the complexity of the symptom.

For its control, we consider that the measures used in conventional clinical practice are not sufficient, but that rehabilitation strategies must be implemented whose objective is the re-education of the individual in their activities of daily living (ADLs), not exclusively oriented to the management of the symptom, since the action on the improvement of the symptoms does not achieve in the majority of the patients a functional improvement of the same nor their readjustment to their daily activity [5].

Most patients perceive this symptomatology as a limiting factor beyond their control that leads them to perform avoidance behaviors that further increase their inactivity, negatively impacting their functionality [6].

The patients with respiratory problems adapting to their symptoms by decreasing the level of activity. This favors a worsening of the physical state and the dyspnea of effort. Dyspnea leads to a progressive disability with loss of mobility, self-esteem, work and social relationships [7].

With these references 8 on the consequences of respiratory pathology, it is evident that the vital capacity of the individual decreases, causing a limitation in their occupational performance. That is why we propose an intervention from a more rehabilitative perspective, in which professionals from different areas of health care have a place, including occupational therapist, forming an interdisciplinary action team.

The benefits of occupational therapy will be appreciated in [8].

1. The quality of life and the general well-being of the individual.

2. The general state of health, both related to respiratory symptoms and tolerance to present exercise.

3. Independence, the patient increases his autonomy and consequently his feeling of self- worth .

4. The ability to play their activities of daily living in society.

Taking into account the incidence data and the improvement in terms of survival in this type of pathologies, we believe it is important to emphasize the control of this symptomatology secondary to these processes, due to its impact on the levels of dependence of individuals. That is why we propose a study that looks at the evaluation of the latter, with special emphasis on the degree of realization of activities of daily life, both the most basic (self-care), and instrumental, since the achievement of both will be which rigorously determines its level of functionality.

\section{Objectives}

- Evaluate the level of dependence of cancer patients in active treatment with respiratory symptoms. 


\section{Annals of Physiotherapy \& Occupational Therapy}

- Evaluate the level of realization of basic activities of daily living (ADLs) in cancer patients under active treatment with respiratory symptoms.

- Evaluate the level of performance of instrumental activities of daily living (ADLs) in cancer patients under active treatment.

- Analyze the impact of gender in the performance of instrumental activities of the daily life of cancer patients.

- Study the correlation between instrumental activities and the daily life performance of cancer patients.

- Establish whether there is a difference in the degree of functionality of cancer patients in their pathological diagnosis.

\section{Methods}

Design: To meet the objectives, a prospective crosssectional observational study with non-probabilistic sampling of accidental assignment will be carried out.

Scope of the Study: Medical oncology service of the University Assistance Complex of Salamanca (CAUSA).

Population and Sample Assignment: The sample consists of 180 patients from the Medical Oncology Service of the University Hospital of Salamanca and being recruited for the study of the following inclusion and exclusion criteria:

\section{Inclusion Criteria}

- Have an anatomopathological diagnosis of cancer disease.

- Be over 18 years old.

\section{Exclusion Criteria}

- Present a level of dyspnea less than 2 on the Medical Research Council (MRC) scale.

\section{Withdrawal Criteria}

- Do not fill in any of the self-administered records.

\section{Procedure}

After the referral of the patients to the occupational therapy team of the service, the subjects that met the inclusion criteria to participate in the study were verified. Subsequently and after obtaining the informed consent signed by the subjects, the evaluation was carried out. For the realization of this, he used, on the one hand, the
Lawton-Brody scale and the Barthel index. In addition, a specific record sheet was designed for the collection of the different variables under study.

\section{Description of Study Variables}

- Age

- Gender

- Pathological diagnosis.

\section{Evaluation Instruments}

- Barthel Index: Measure of physical disability with proven validity and reliability, easy to apply and interpret and whose routine use is recommended. Useful for assessing functional disability in the ADLs basic.

- Lawton and Brody scale: The Lawton and Brody scale is one of the most widely used ADLs instrumental measurement instruments internationally and the most used in geriatrics units in Spain. It values 8 items and assigns them a numerical value 1 (independent) or 0 (dependent). Information is obtained by directly asking the individual or their primary caregiver. It has proven useful as an objective and brief assessment method that allows the implantation and evaluation of a therapeutic plan both at the level of the daily care of the patients and at the teaching and research level. Very useful scale to detect the first signs of deterioration of the elderly.

\section{Statistical Methodology}

\section{Descriptive Statistics}

- Quantitative variables that follow a normal distribution are defined by mean and standard deviation.

- For quantitative variables that do not follow this distribution, the median is used instead of the mean as a measure of centralization.

\section{Analytical Statistics}

- To analyze two quantitative variables, Pearson's correlation coefficient will be used.

- To evaluate two qualitative variables, the CHI-SQUARE statistical test will be used.

- To define a qualitative variable (more than two categories) and a quantitative variable, the ANOVA statistical test is used, whose graphical representation is carried out using diagrams.

\section{Data Processing}

The data analysis process was carried out with the statistical program SPSS version 24.0 


\section{Results}

The results corresponding to the descriptive part of the study of the different variables analyzed can be seen in Table 1 . And Figure $1 \& 2$. The total sample of the study was 180 individuals. The average age of the sample was $68.48( \pm 10,513)$ years. As for the gender of the participants, the $52.8 \%$ of the sample were male, $47.2 \%$ for women (85 women and 95 men). Regarding pathologic diagnosis the $22,8 \%$ had a diagnosis of breast a $31.7 \%$ lung and one $45.6 \%$ had tumors related to the digestive system [9].

\begin{tabular}{|l|c|c|c|c|c|c|}
\hline \multicolumn{2}{|c|}{ Gender } & \multicolumn{2}{|c|}{ Age } & Anatomopathological Diagnosis & \\
\hline $\mathrm{N}(180)$ & Half & $\mathrm{S}$ & Breast Pathology & $\mathrm{N}=41$ & $22.80 \%$ \\
\hline Male & $95(52.8 \%)$ & 68.48 & 10,513 & Pulmonary & $\mathrm{N}=57$ & $31.70 \%$ \\
\hline Woman & $85(47.2 \%)$ & & & A. Digestive & $\mathrm{N}=82$ & $45.60 \%$ \\
\hline
\end{tabular}

Table 1: Descriptive results intervening variables.

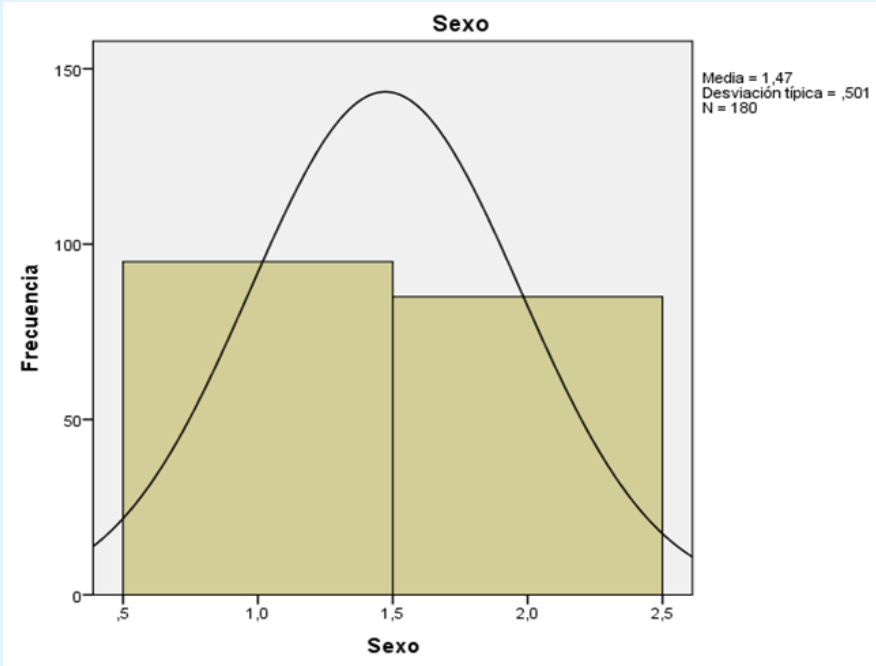

Figure 1: Graphical representation of the variable "gender" in the study.

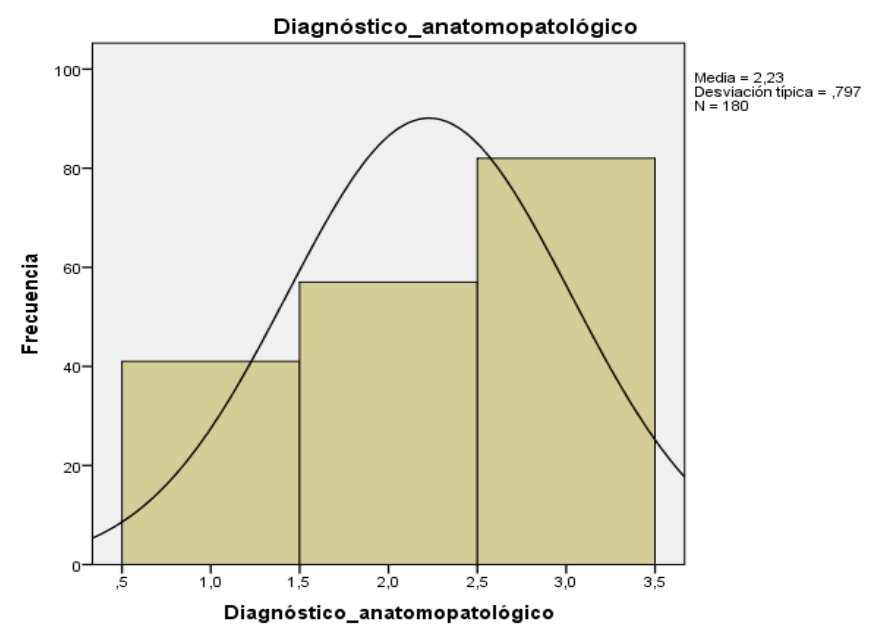

Figure 2: Graphical representation of the variable "pathological diagnosis" in the study. 


\section{Annals of Physiotherapy \& Occupational Therapy}

Regarding the results obtained related to the level of dependence, we observed in the Lawton and Brody scale, an average score of $4.31( \pm 1,832)$ points, which is identified as moderate levels of dependence; while, if attend to the evaluation of the basic activities of daily life, measured with the Barthel index, have an average score of $42.53( \pm 2,789)$ points, that is to say a moderate level of dependence (Table 2). Being individuals with an anatomic diagnosis of lung disease who have worse scores.

\begin{tabular}{|c|c|c|c|}
\hline \multicolumn{2}{|c|}{ Barthel Index } & \multicolumn{2}{c|}{ Lawton_Brody Scale } \\
\hline HALF & $S$ & HALF & $S$ \\
\hline 42.53 & 2,789 & 4.31 & 1,832 \\
\hline $\begin{array}{c}\text { Moderate Dependency Level } \\
\text { (Abvd) }\end{array}$ & $\begin{array}{c}\text { Moderate Dependency Level } \\
\text { (Aivd) }\end{array}$ \\
\hline
\end{tabular}

Table 2: Variable descriptive results to study.

Regarding the correlations, observed, on the one hand, a strong and statistically significant correlation between the Lawton and Brody scale scores and those of the Barthel index ( $r=0.803$, for $p<0.05$ ), on the other, obtained A significant correlation $(\mathrm{r}=-0.403$, for $\mathrm{p}$ $<0.001$ ) between age and the performance of instrumental activities of daily life, the older one correlates with a higher level of dependence on individuals (Table 3).

\begin{tabular}{|c|c|}
\hline Correlations & Lawton Brody \\
\hline Barthel & $\mathrm{r}=0.803(\mathrm{p}<0.05)^{*}$ \\
\hline Age & $\mathrm{r}=-0.403(\mathrm{p}<0.001)^{*}$ \\
\hline
\end{tabular}

Table 3: Correlation between variables under study.

* Pearson correlation

On the contrary, no significant relationship can be established between the gender of the individuals and the levels of dependence expressed.

\section{Discussion}

The main objective of the study was to assess the level of dependence of cancer patients in active treatment with respiratory symptoms. We seek to know more accurately what was the current situation of patients who are in active treatment. In recent years we are aware of the significant increase in survival in cancer patients [10], but linked to this increase in survival we must know what the situation is in terms of quality of life related to their health, and according to the scientific literature we observe that one of the largest and most accurate indicators of this is the level of dependence expressed by patients [11].

In this aspect we have found that a moderate dependence both in terms of performing basic and instrumental activities of daily life. This is directly related to what was expressed in previous studies by authors such as Vockins H, Purcell A, Lehmann JF, et al. [12-14].

In addition, not only the increase in survival can contribute to a change in the self-worth of these individuals, but in many cases the increase in treatment lines is shown to lead to side effects such as tumor asthenia, chemobrain or respiratory problems $[15,16]$. In the latter the scientific literature considers them as one of the biggest culprits of functional impairment of individuals, studies such as Kealey P, Holm LV, et al. $[17,18]$.

Another aspect to consider would be the age of the patients, since, according to the existing scientific literature, there is a clear consensus on the negative impact that age has in terms of loss of autonomy. We wanted to check if this also affected cancer patients and we observed how there was a statistically significant correlation, with a degree of significance $p<0.001$ if we talked about performing AIVD. While, in the case of the ABVD, we cannot establish a direct relationship. In this regard, we emphasize, that many studies speak of a significant relationship, as long as they speak of individuals in a more advanced stage of oncologic disease, which can make us indicate that diminish in the AIVD is greater in this type of patients that in the normality of people without oncological pathologies. In addition, we believe it is appropriate to continue studying and deepening the subject, since more conclusions can be drawn from the study of patients with a more advanced stage, even from the study of palliative patients.

In addition, we consider it important in future studies, to quantify more accurately, once we have known that there is a clinical deterioration in cancer patients with respiratory pathology, the importance of this respiratory clinical deterioration induced by oncological pathology or its treatments in the loss of patient autonomy. Quantifying the degree of respiratory involvement and correlating it with functional loss, in order to know everything more accurately.

In this sense, we believe it is important to be able to include the figure of the occupational therapist for the treatment of these patients from 


\section{Annals of Physiotherapy \& Occupational Therapy}

a rehabilitative approach, thanks to which the functional clinical deterioration can be counteracted. Considering important, not only, the hospital intervention, but a subsequent follow-up at home to be able to extrapolate everything learned in a real context.

\section{Conclusion}

- Oncological patients in active treatment have moderate levels of dependence in carrying out their activities of daily living.

- Strong correlation between the levels of performance of instrumental and basic activities of daily life in cancer patients under active treatment.

- Older cancer patients have higher levels of dependence.

- There is no relationship between the gender of cancer patients and the performance of instrumental activities of daily living.

- Oncological patients with a pathological diagnosis of lung cancer had the worst levels of dependence.

\section{References}

1. National Comprehensive Cancer Network (2013) Clinical Practice Guidelines in Oncology: Cancerrelated-fatigue.

2. Horneber M, Fischer I, Dimeo F, Ulrich JU, Weiss J, et al. (2012) Cancer related fatigue: epidemiology, pathogenesis, diagnosis and treatment. Dtsch Arztebl Int 109(9): 161-171.

3. Reuben DB, Rubenstein LV, Hirsch SH, Hays RD (1992) Value of functional status as a predictor of mortality: Results of a prospective study. Am J Med 93(6): 663-669.

4. American Occupational Therapy Association (2015) Standards of practice for occupational therapy.

5. Hewitt M, Rowland JH, Yancik R (2013) Cancer survivors in the United States: Age, health, and disability. J Gerontol A Biol Sci Med Sci 58(1): 82-91.

6. Kuroi K, Shimozuma K (2004) Neurotoxicity of taxanes: Symptoms and quality of life assessment. Breast Cancer 11(1): 92-99.

7. Clark F, Azen SP, Carlson M, Mandel D, LaBree L, et al. (2001) Embedding health-promoting changes into the daily lives of independent-living older adults: Long-term follow up of occupational therapy intervention. J Gerontol B Psychol Sci Soc Sci 56(1): 60-63.

8. Sanchez F, Barco E (2008) Continuous oncology care: control of more prevalent symptoms: emesis and other digestive complications, asthenia, cachexia, other symptoms. End-of-life care. Grief In: Cruz JJ, Rodriguez CA, Barco E Clinical Oncology $4^{\text {th }}$ (Ed.) Nova, Sidonia, Madrid, pp: 585.

9. Cooper J (2007) Challenges faced by occupational therapist in oncology and palliative care. $2^{\text {nd }}(\mathrm{Ed}$.), jhon Wiley, Londre, pp: 284.

10. Williams GR, Deal AM, Nyrop KA, Pergolotti M, Guerard EJ, et al. (2015) Geriatric assessment as an aide to understanding falls in older adults with cancer. Support Care Cancer 23(8): 2273-2280.

11. Curt GA, Breitbart W, Cella D, Groopman JE, Horning SJ, et al. (2000) Impact of cancer-related fatigue on the lives of patients: New findings from the Fatigue Coalition. The Oncologist 5(5): 353-360.

12. Vockins H (2004) Occupational therapy intervention with patients with breast cancer: A survey. Eur J Cancer Care 13(1): 45-52.

13. Purcell A Fleming J, Bennett S, Haines T (2010) Development of an educational intervention for cancer related fatigue. Br J Occup Ther 73(7): 327333.

14. Lehmann JF, DeLisa JA, Warren CG, deLateur BJ, Bryant PL, et al. (1978) Cancer rehabilitation: Assessment of need, development, and evaluation of a model of care. Arch Phys Med Rehabil 59(9): 410419.

15. Ross L, Petersen MA, Johnsen AT, Lundstrøm LH, Groenvold M, et al. (2012) Are different groups of cancer patients offered rehabilitation to the same extent? A report from the population-based study "The Cancer Patient's World". Support Care Cancer 20(5): 1089-1100.

16. Halkett GK, Ciccarelli M, Keesing S, Aoun S (2010) Occupational therapy in palliative care: Is it underutilized in Western Australia? Aust Occup Ther J 57(5): 301-309.

17. Kealey P, McIntyre I (2005) An evaluation of the domiciliary occupational therapy service in palliative 


\section{Annals of Physiotherapy \& Occupational Therapy}

cancer care in a community trust: A patient and carers perspective. Eur J Cancer Care 14(3): 232-243.

18. Holm LV, Hansen DG, Johansen C, Vedsted P, Larsen PV, et al. (2012) Participation in cancer rehabilitation and unmet needs: A population-based cohort study. Support Care Cancer 20(11): 2913-2924. 